\title{
Ley 1580 del 2012: pensión familiar para la protección de la vejez*
}

\section{Law 1580, 2012: family pension to protect oldness}

\author{
Gloria Amparo Triviño Buitrago
}

Economista, Especialista en Gerencia, correo electrónico Gatbgat@Hotmail.com, correspondencia calle 21 no 16 ñ40 local B Armenia Quindío Colombia

Cómo citar: Tiviño, G.A (2016). Ley 1580 del 2012: pensión familiar para la protección de la vejez. Inciso 18 (1) 41-49.

doi: http://dx.doi.org/10.18634/incj.18v.1i.473

Recibido:11/11/2015 Revisado: 15/01/2016 Aceptado: 15/06/2016

\section{Resumen}

Este artículo pretende demostrar por qué la Ley 1580 del 2012 no es una norma que conserva el principio de igualdad constitucional en Colombia para el cubrimiento y protección de la vejez. En él se muestra que el sistema pensional tiene una cobertura muy baja y ley analizada discrimina a la clase trabajadora de los niveles altos del sistema para identificación de beneficios (Sisben) en el Régimen de Prima Media

Palabras clave: Subsidios-grupo poblacional. igualdad social, sistema de selección de beneficiarios para programas sociales, salario mínimo, clase trabajadora

\begin{abstract}
The purpose of this article is to demonstrate that Law 1580, 2012 is not a rule that keeps the principle of constitutional equality in Colombia for coverage and protection of oldness. This article shows that the pension system has a very low coverage, and that the analyzed law discriminates the worker class of high levels of the system for identification of benefits (SISBEN) in the regime of Mean Bonus.
\end{abstract}

Keywords: Subsidies-population group. Social equality, Beneficiary selection system for social programs, minimal salary, worker class.

\footnotetext{
*Trabajo de investigación derivado de la tesis de posgrado de la Especialización en Gerencia.
} 


\section{Introducción}

En la sociedad colombiana, el Estado se enfrenta a un gran reto de asegurabilidad y protección de los adultos mayores, los cuales, podemos decir, son los menos amparados.

Para mitigar esta problemática el Estado colombiano ha tratado de crear nuevas formas de protección, como subsidios económicos, en salud, diferentes programas de apoyo y un Sistema de Identificación y Clasificación de Potenciales Beneficiarios para Programas Sociales (en adelante Sisben), en pocas palabras una estratificación. El tema específico de esta investigación es la Ley 1580 de 2012, también llamada pensión familiar, diseñada por el gobierno para que la vejez sea protegida de alguna manera. Se basa en la cotización hecha en pensión por dos esposos o compañeros permanentes que no alcanzaron cada de uno de manera individual a completar los requisitos de semanas para la pensión de vejez, según la normatividad vigente en Colombia.

El problema que aborda este artículo es el siguiente: ¿La Ley 1580 del 2012 conserva el principio de la igualdad constitucional en el sistema general de pensiones colombiano?

Se sostiene que la Ley 1580 del 2012 no conserva el principio de igualdad constitucional en Colombia, porque a muchos ciudadanos se les pretende materializar sus derechos por medio de leyes y normas que no son bien estructuradas, realizadas con estudios poco profundos, en una búsqueda de una forma rápida para generar una mayor cobertura.

El sistema general de pensiones en Colombia no tiene una cobertura total para los colombianos que no reúnen los requisitos de edad y semanas para completar el derecho a una pensión así sea de salario mínimo, por lo tanto, no hay una protección completa a la vejez. Es por ello que la pensión familiar nace debido a la gran preocupación que tiene el Gobierno Nacional por la escasa cobertura pensional, la desigualdad del sistema y la falta de recursos económicos para que este se pueda sostener. Actualmente se están haciendo los mayores esfuerzos para que los trabajadores de Colombia cuenten con un ingreso de por lo menos el salario mínimo a la edad de retiro.
Colpensiones es una entidad oficial encargada de administrar un fondo de cotización pensional con aportes del Estado, con un área encargada de servicio al ciudadano (puntos de atención) donde se radican todo tipo de solicitudes y a su vez se realizan las asesorías del Régimen de Prima Media con prestación definida que administra la entidad. Desde el inicio del año 2015 se atiende a las personas que creen cumplir con los requisitos que exige la Ley 1580 del 2012 de Pensión Familiar, además se realiza consultoría con los agentes de servicio de la entidad yse identifica que son muy pocas la personas que pueden acceder al reconocimiento de esta prestación económica.

Debido a que no todas las personas poseen Sisben del nivel $1 \mathrm{o} 2$, requisito indispensable para dicho reconocimiento, quienes más demandan la pensión familiar son personas de la clase trabajadora del estrato 3 y 4 con un buen número de semanas cotizadas, pero, por lo expuesto, no tendrían derecho a ella.

Es por ello que la norma no está bien estructurada desde el punto de vista social, porque se están excluyendo muchos colombianos que podrían obtener por lo menos un salario mínimo legal vigente en sus hogares. Actualmente, existe un $57 \%$ de personas sin cobertura, la norma podría ser más flexible buscando disminuir este porcentaje.

El tipo de estudio realizado en este artículo es de carácter cualitativo, de alcance descriptivo. La información que se va a utilizar en esta investigación fue extraída de revistas de opinión, artículos de los diarios colombianos, Banco de la República, leyes y decretos referentes al tema de seguridad social.

\section{Colombia tiene una cobertura muy baja para la protección de la vejez}

En el año 1946 nace el sistema de seguridad pensional en Colombia, en el cual se crea el primer régimen pensional y la aparición de una serie de cajas de previsión a nivel nacional, departamental y local, pero solamente para el sector público.

En 1967 las pensiones de los trabajadores del sector privado se reglamentaron y las declaran obligatorias y delegan esta administración al Instituto Colombiano 
de los Seguros Sociales (ICSS) donde el modelo que se creó en pensiones era el de prima media, en que los ciudadanos en edad de trabajar realizaban sus aportes a un fondo común, del cual se pagaban las mesadas a las personas que tuvieran el derecho a pensión.

La formalización del sistema de seguridad social en pensión en cuanto a los beneficios que tenía hasta ese momento, después de mucho tiempo de funcionamiento se empezó a ver con algunas dificultades, según informe de Fedesarrollo de abril de 2010se identificaron algunos factores:

Si bien la implementación del Régimen de prima media trajo beneficios a una parte de la población y creo las bases para el desarrollo del sistema pensional, después de veinte años de funcionamiento el esquema comenzó a mostrar señales de insostenibilidad financiera, baja cobertura e inequidad originadas principalmente en cinco factores:(i) la tasa de cotización no se incrementó gradualmente como se había previsto desde el principio; (ii) el Estado incumplió su parte de la cotización; (iii) los excesivos beneficios, relativos a los aportes; (iv) la existencia de una amplia gama de regímenes especiales y de cajas administradoras; y (v) el cambio demográfico, que implicó menores aportes (cada vez menos jóvenes) y mayores gastos (la gente vivía más años). (Fedesarrollo, 2010: 5)

Es así como se observa que el sistema se ha debilitado y llevó a que se marcara desde un inicio la insostenibilidad del régimen administrado por el Instituto del Seguro Social. La Ley 100 de 1993 nace como alternativa para aumentar la cobertura pensional, especialmente para los más vulnerables, dándoles la posibilidad de realizar cotizaciones al sistema pensional como independientes y en el régimen subsidiado en cotización, pero la baja cobertura que se ha dado a medida que pasa el tiempo paradójicamente está afectando directamente a la población menos favorecida económicamente, donde lo único que ha hecho es que los gobiernos se focalicen en entregar subsidios a la población informal, lo cual desincentiva la formalidad y la afiliación a la seguridad social.

Es por ello que se muestra que la informalidad y el desempleo afectan considerablemente a los jóvenes, a los trabajadores menos calificados y a los independientes, quienes tienen menos cobertura en pensión. Después de 20 años el sistema pensional colombiano sigue presentando los mismos problemas estructurales, como la baja cobertura, el déficit fiscal, la inequidad.

El gobierno proteccionista ha hecho grandes esfuerzos a través de diferentes reformas y un número importante de fallos de la Corte Constitucional que en estricto sentido han legislado en materia trascendental para el sistema pensional, pero todo este empeño no ha sido suficiente, se requiere cambios de mayor envergadura a los ya acometidos, como es el compromiso entre el estado, el sector industrial, las agremiaciones de trabajadores y las administradoras de pensiones para hacer un trabajo más a fondo, donde estén interviniendo todas las partes que puedan entregar las herramientas y proyectos que logren ser aplicados para el aumento de la cobertura en la seguridad social Colombiana.

En Colombia hay 22 millones de trabajadores, de los cuales 7.7 millones cotizan o ahorran activamente en el Sistema General de Pensiones, pero de ellos solamente 1.6 millones recibirán una pensión al momento de su retiro. El 90 por ciento se queda en el camino porque no ha ahorrado lo suficiente o no han trabajado lo suficiente. (Ministerio del Trabajo, 2015)

El Ministerio de Trabajo en la presentación del nuevo modelo de protección para la vejez hace una comparación integral donde la cobertura de Colombia en comparación con otros países del continente americano es solamente del $32.8 \%$, la cual es muy inferior y concluye que la principal causa para que esto ocurra es la informalidad laboral (Ministerio del trabajo, marzo de 2015)

El país no puede seguir produciendo economías informales y seguir cargando a los empresarios de una cantidad de impuestos, donde lo único que se hace es limitar la generación de empleo que se lograría ofertar en los diferentes sectores económicos que existen en el país y podría ir en aumento las vacantes laborales, mientras que lo único que realizan por otro lado, creyendo que es la mejor solución, es subsidiar 
el empleo informal y alimentarlo aún más para que se extienda y tenga menos posibilidades de legalizarse, llevando a sus integrantes a estar cada vez más restringidos económicamente, impidiéndoles cotizar el sistema general de seguridad social

El porcentaje bajo de cobertura se debe también a que los empresarios generadores de empleo formal tienen el problema de corrupción de los dirigentes de este país; miles de millones de pesos del gasto público destinados a incentivos para las empresas son desviados a manos inescrupulosas que no administran de manera transparente los recursos y estos se terminan perdiendo o convirtiéndose en gasto privado, dichos dineros no llegan a suplir las necesidades de seguridad social que existen actualmente en el país, lo que impide que Colombia pueda mejorar de manera más radical la calidad de vida de los ciudadanos, generando simplemente un desestímulo al empleo formal.

\section{Ley 1580 del 2012 como alternativa para aumentar la cobertura}

En los últimos años en Colombia el Estado ha estado trabajando de la mano del Departamento Nacional de Planeación, con el objetivo de revisar y diseñar posibles soluciones a distintas problemáticas económicas y sociales, de ahí que:

El Plan Nacional de Desarrollo "Prosperidad para Todos" 2010-2014 plantea que el país requiere un Sistema de Protección para la Vejez (SPV) universal, incluyente y equitativo, que busque aumentar gradualmente la cobertura que tienen los mecanismos vigentes de protección a la vejez y lograr que cada vez más personas mejoren sus condiciones de vida al final de su ciclo productivo, a través del esfuerzo personal y esquemas solidarios definidos por la Ley. (DNP, 2012: 3)

Debido a lo anterior y en con el objetivo de evitar un problema socioeconómico mayor, el Gobierno Nacional a través del Ministerio del Trabajo ha venido socializando con los diferentes grupos económicos, sociales, laborales e institucionales el nuevo esquema de protección a la vejez.
Esta propuesta es para el Gobierno la solución a la incertidumbre generada por un alto porcentaje de colombianos que se encuentran desprotegidos, que llegan a la edad de pensionarse sin contar con un ingreso que le permita vivir una vejez digna y su último ciclo de vida con una entrada económica con la cual pueda atender sus necesidades básicas de alimentación, vivienda, salud y recreación. En pleno siglo XXI nadie debería tener problemas de falta de cobertura a la vejez, pero el sistema como tal lo permite, por las deficiencias de coyuntura económica, política, institucional y social que presenta el país.

El modelo de protección de la vejez es una iniciativa que busca subsanar la poca cobertura pensional, la desigualdad del sistema, la falta de garantías y solidez de tipo fiscal para evitar su insostenibilidad, pero, ¿cómo hacerlo?, es el interrogante que el Gobierno Nacional mantiene, por eso dentro del modelo propone los siguientes esquemas y leyes:

1. Consorcio Colombia Mayor en dos frentes: subsidios al adulto mayor y subsidios a la cotización en pensión.

2. Beneficios económicos periódicos (BEPS).

3. Cotización retroactiva.

4. Pensión Familiar Ley 1580 De 2012.

5. Flexibilización Fondo de Garantía de Pensión Mínima.

Este nuevo modelo de protección a la vejez busca mantener la edad y las semanas mínimas para acceder a la pensión de vejez. La Ley 1580 de 2015 tiene como pilar fundamental que dos integrantes de una misma familia (esposos o compañeros permanentes) puedan acceder a un ingreso mínimo, es decir, una pensión del salario mínimo para un núcleo familiar conformado por dos personas que sostienen un vínculo afectivo, económico y marital, solución que aumentaría la cobertura para la vejez por familia.

Por lo tanto, la Pensión Familiar como alternativa para aquellas personas que de otra forma no pueden acceder a un ingreso en su vejez, puede dar alivio en el corto plazo para un sistema que no ha logrado aún 
la cobertura deseada, pero no deja de ser una solución parcial que no ataca la problemática de fondo por los requisitos que se le exige a los esposos; porque el derecho fundamental al que se tiene a la seguridad social debe tener un verdadero aumento en la cobertura generando así diferencias discriminatorias entre parejas similares que en conjunto logran acumular semanas cotizadas necesarias para acceder a la pensión familiar, pero, por una clasificación no equitativa del Sisben, se les termina negando un derecho.

Se hace necesario implementar un modelo que ayude a subsanar la poca cobertura existente y mediante este esquema encaminar esfuerzos para que todos los trabajadores formales e informales cuenten con un ingreso al llegar a sus edades de retiro ya sea dentro del sistema pensional, el cual es el ideal al que tenga derecho la gran mayoría de colombianos, o por medio de una ayuda del gobierno para que no queden sin protección alguna. A continuación, se revisan las políticas públicas sobre este tema de suma importancia.

\section{Clase trabajadora discriminada con la Ley 1580}

En la creación de la Ley 1580 del 1 de octubre del 2012 se tuvo en cuenta la puntuación del Sisben para poder clasificar las personas y así asignar una categoría.

El Sisben es un instrumento creado por el estado que sirve para identificar los hogares, las familias o los individuos menos favorecidos económicamente y vulnerables como potenciales beneficiarios de programas sociales, entre los cuales se encuentra la afiliación en salud al régimen subsidiado. El Sisben permite establecer el grado de pobreza de los hogares, con el propósito de focalizar (asignar) recursos de inversión social; no solo se utiliza para reconocer a los beneficiarios del régimen subsidiado en salud, puede ser usado por cualquier programa social que requiera clasificara las personas según sus condiciones de vida para ser beneficiarios de planes y programas diseñados por el gobierno nacional para los más pobres.
Según mencionado, el Estado pretende cubrir a los menos favorecidos económicamente y por tal motivo, estratifica los beneficiarios de la Ley 1580 de 2012 en su artículo 151:

Pensión Familiar en el Régimen de Prima Media
con Prestación Definida. Quienes cumplan
los requisitos para adquirir el derecho a la
indemnización sustitutiva de la pensión de vejez en
el sistema de prima media con prestación definida,
podrán optar por la pensión familiar, cuando los
dos cónyuges o compañeros permanentes obtengan
la edad mínima de jubilación y la suma del número
de semanas de cotización supere el mínimo de
semanas requeridas para el reconocimiento de la
pensión de vejez.

k) Solo podrán ser beneficiarios de la Pensión Familiar, en el Régimen de Prima Media, aquellas personas que se encuentren clasificadas en el Sisbén en los niveles 1, 2 y/o en cualquier otro sistema equivalente que diseñe el Gobierno Nacional. (Ley 1580, 2012)

Con lo expuesto en el literal $\mathrm{k}$, los eventuales beneficiarios de la pensión familiar afiliados al régimen de prima media, pueden ser los clasificados en el nivel 1y 2; allí se da una diferencia entre los que están en el grupo que aplica y quienes no lo están. Pueden existir dos parejas con los mismos ingresos y con la misma cantidad de semanas de cotización en su historia laboral, pero se limita el acceso a esta pensión a quienes no cumplen con el requisito de Sisben. Aunque hagan parte de estratos económicos más altos, también se encuentran en condiciones de vida insatisfactorias, ya que no pueden suplir sus necesidades básicas. Es por ello que la clasificación del Sisben no puede ser un determinante para acceder a este tipo de pensión, dado que desconoce que la pensión de vejez en el régimen de prima media ha estado históricamente determinada por edad y semanas de cotización, mientras que la ley de pensión familiar genera discriminaciones injustificadas, porque todos deben tener el mismo derecho.

Todos los ciudadanos colombianos deben tener una clasificación en el Sisben del nivel 1 al 6, y lo único que esto genera es poner una limitante formal para desconocer derechos pensiónales a familias que en algún momento acumularon semanas y hacían parte 
de la clase trabajadora de Colombia, ubicados en otros estratos socioeconómicos como profesionales y técnicos que trabajaron en empresas del país que cuentan con semanas y edad pero se les desconoce la pensión porque están en niveles 3,4,5 o 6 donde son categorías que también evidencian que se trata de personas que pertenecen a población pobre de nuestro país y no cuentan con recursos económicos así tengan esa estratificación.

Hoy día se encuentran excluidas por el sistema y no tendrán derecho a una pensión que les permita una vejez digna, es por ello que esta ley afecta fundamentalmente a personas que se encuentran en condiciones de debilidad, a grupos marginados o discriminados, a sectores sin acceso, sin toma de decisiones o minorías discretas, lo anterior resulta evidente si se observa que muchas personas pueden ser destinatarias para aplicar este tipo de pensión en el régimen de prima media donde lo único que está haciendo es limitar exageradamente la posibilidad del derecho fundamental a la seguridad social para que tenga un verdadero aumento de cobertura, porque por una clasificación no justa del Sisben se les estén negando un derecho irrenunciable, el cual debe estar generado por una edad y semanas de cotización, en un privilegio que surge de una clasificación que está dirigida a focalizar el sistema subsidiado de pensiones y no a limitar el acceso a los beneficios que nacen del sistema contributivo, donde esta norma tiende a ser objeto de discriminaciones subjetivas y desproporcionadas para las personas que están en este proceso.

\section{La pensión familiar y el principio de igualdad}

La clase trabajadora colombiana que se encuentra clasificada en los niveles de Ssben del 3 hasta el 6 que pueden cumplir con los requisitos de edad y semanas cotizadas pero no la categoría del 1 y 2 , limita considerablemente la posibilidad de que trabajadores que en algún momento tuvieron la oportunidad de trabajar y cotizar en el tema de seguridad social, garantía entregada en el del artículo 48 de la Constitución Política donde dice: "Se garantiza a todos los habitantes el derecho irrenunciable a la Seguridad Social" (Const., 1991, párr. 1).
El Estado, con la participación de los particulares, ampliará progresivamente la cobertura de la Seguridad Social que comprenderá la prestación de los servicios en la forma que determine la Ley.

Seguridad Social podrá ser prestada por entidades públicas o privadas, de conformidad con la ley. No se podrán destinar ni utilizar los recursos de las instituciones de la Seguridad Social para fines diferentes a ella, teniendo en cuenta que es un servicio público de carácter obligatorio que se debe prestar bajo la dirección, coordinación y control del Estado buscando la eficiencia, universalidad y solidaridad.

La ley definirá los medios para que los recursos destinados a pensiones mantengan su poder adquisitivo constante y se pueda cumplir con las obligaciones del sistema en cuanto al pago de las mesadas pensionales.

Por lo tanto, se puede decir que en un grupo considerable de ciudadanos y trabajadores de este país, se le está atentando contra sus derechos para poder adquirir un ingreso por lo menos del salario mínimo legal vigente para suplir sus necesidades básicas del adulto mayor, entiéndase por esta palabra la total satisfacción en lo que se refiere a la protección, promoción y defensa de los derechos de las personas de la tercera edad para que vivan dignamente, los cuales están respaldados en la Ley 1251 del 2008 en los artículos 1 y 4 donde se habla de toda la protección de las personas de más de 60 años donde se les está limitando a este derecho a colombianos que pueden acceder por medio de la pensión familiar, lo cual no ayuda a una ampliación de la cobertura del modelo de protección de la vejez que se está aplicando en Colombia. La Constitución Política de Colombia reza en su artículo 13:

Artículo 13. Todas las personas nacen libres e iguales ante la ley, recibirán la misma protección y trato de las autoridades y gozarán de los mismos derechos, libertades y oportunidades sin ninguna discriminación por razones de sexo, raza, origen nacional o familiar, lengua, religión, opinión política o filosófica. (Const., 1991: Art 13)

Así las cosas, vemos claramente que el principio de igualdad está consagrado en el titulo 2 de los derechos, las garantías y los deberes, capítulo $1 \mathrm{de}$ 
los derechos fundamentales, ósea es un derecho fundamental en Colombia. La Sentencia T-098/94 de la Corte Constitucional en el uno de sus apartes indica:

[...] La igualdad, en sus múltiples manifestaciones - igualdad ante la ley, igualdad de trato, igualdad de oportunidades -, es un derecho fundamental de cuyo respeto depende la dignidad y la realización de la persona humana. Las normas que otorgan beneficios, imponen cargas u ocasionan perjuicios a personas o grupos de personas de manera diversificada e infundada contrarían el sentido de la justicia y del respeto que toda persona merece. La discriminación, en su doble acepción de acto o resultado, implica la violación del derecho a la igualdad. Su prohibición constitucional va dirigida a impedir que se coarte, restrinja o excluya el ejercicio de los derechos y libertades de una o varias personas, se les niegue el acceso a un beneficio o se otorgue un privilegio sólo a algunas, sin que para ello exista justificación objetiva y razonable. (Corte Constitucional, 1994: párr. 4)

Aunado con el principio de igualdad, el profesor Martín Leonardo Suárez Varón, en su libro titulado El principio de igualdad en la Constitución y la jurisprudencia (2006), explica en qué circunstancias debemos tener claridad sobre este aspecto y por qué es adoptado por el legislador colombiano para ser un derecho fundamental. Vemos a lo largo del tiempo y con la aplicación de cada normatividad, que dicho principio cada vez es dejado a un lado, ya que las normas van siempre ligadas a la completitud de ciertos requisitos diseñados por el legislador sin tener en cuenta en la mayoría de las circunstancias la violación de este principio y la afectación que eso conlleva para diferentes ciudadanos que no puede cumplir con los requisitos impuestos.

Podemos aseverar entonces que la pensión familiar establecida en este país parte de una serie de medidas desesperadas que buscan incrementar la cobertura del sistema contributivo de la protección social. Contiene problemas de diseño en algunos puntos, como el tema del Sisben para el régimen de prima media con prestación definida, que la llevan a que sea una ley con aspectos de institucionalidad y en otros sea inequitativa.
Aunque este tipo de pensión es un derecho, no hay lugar que este régimen se favorezca solamente a las personas de población más pobre. Es cierto que los grupos familiares clasificados en los niveles 1 y 2 del Sisben por su situación de vulnerabilidad desde la parte socioeconómica merecen especial protección del Estado, pero insuficiente para concluir que personas clasificadas en otros niveles no requieran también de total protección. La esencia del proyecto de ley que dio lugar a la Ley 1580 era que todas las parejas colombianas pudieran acceder a la pensión familiar, lo cual no se aplica actualmente. De acuerdo con lo anterior, se puede deducir que en cuanto al sistema pensional se debe buscar que al llegar a la vejez y tener dificultades para trabajar, se debe recibir un salario justo para mantener los niveles de vida. Por equidad y solidaridad todas las personas deben contribuir para que se pueda financiar las pensiones.

Nuestro país debe observar, como lo hizo chile en algún momento, que la gran mayoría de las personas que cotizan no tienen la suerte de pensionarse por la problemática estructural de los mercados laborales, donde los contratos de trabajo deben ser más estables, es decir, de periodos de tiempo a largo plazo, como mínimo de un año. También la informalidad que existe, en la que su única salida benéfica es el trabajo formal y así poder que las personas acumulen tiempos de cotización para las mujeres y hombres y con la edad exigida por la ley para obtener su pensión de vejez. De tal modo que no sea necesario tener que buscar la alternativa que se está aplicando actualmente en el país del nuevo modelo de protección a la vejez como es la ley 1580 de 2012, la cual no está bien estructurada en cuanto a sus requisitos y tampoco está acorde con los principios constitucionales de Colombia sobre proteger al ciudadano en materia de seguridad social.

\section{Conclusiones}

La Ley 1580 del 2012 o también llamada pensión familiar se demostró en este artículo que la norma no está bien estructurada desde el punto de vista social, porque se están excluyendo muchos colombianos que podrían obtener por lo menos un salario mínimo legal vigente en sus hogares, donde la norma al ser más flexible podría disminuir la población que esta desprotegida. 
Se puede exponer que la pensión familiar fue establecida en este país por una serie de medidas desesperadas para buscar incrementar la cobertura en el sistema contributivo de la protección social. Ese afán generó problemas de diseño estructural como la aplicación del Sisben para el régimen de prima media con prestación definida administrada por Colpensiones.

Esta ley solamente favorece a las personas de población más pobre o vulnerable que se encuentran clasificados en los niveles 1 y 2 del Sisben, sin embargo, no hay razón para concluir que personas clasificadas en otros niveles superiores no requieran de total protección como sino necesitaran de esta opción para poder percibir un ingreso entre las parejas.

Se puede apuntar que la Ley 1580 del 2012 no es una norma equitativa constitucionalmente para el cubrimiento de la protección de la vejez en Colombia por que a muchos ciudadanos se les pretende materializar sus derechos por medio de leyes y normas que no están bien estructuradas para lograr realmente que la pensión familiar se utilice como una opción más para lograr aumentar la cobertura en las familias según el modelo de la protección de la vejez estructurado por el Gobierno Nacional.

Con el objetivo de aportar a la mejora de la Ley 1580 del 2012,el Gobierno Nacional por medio del Ministerio de la Protección Social debe reconsiderar el tema de la clasificación de los niveles en el Sisben para que sea más equitativa esta propuesta, porque lo único que está ocasionando es limitar derechos pensionales a parejas que en algún momento acumularon semanas y eran trabajadores ubicados en estratos socioeconómicos más altos, pero que actualmente no se encuentran pensionados por vejez y están desprotegidos en su tercera edad.

\section{Referencias bibliográficas}

Constitución Política de Colombia www. procuraduria.gov.co(20161991) Artículo 13 Recuperadodehttp://www.procuraduria.gov. co/guiamp/media/file/Macroproceso $\% 20$ Disciplinario/Constitucion_Politica_de Colombia.htm

Corte Constitucional de Colombia (1994) Derecho a la igualdad. Recuperado de http://www.corteconstitucional.gov.co/ RELATORIA/1994/T-098-94.htm

Dirección Nacional de Planeación, DNP (DNP, 2012, Consejo2012) D Nacional de Politica Económica y Social República de Colombia, documento conpes Conpes 156. Recuperado de https://colaboracion.dnp.gov.co/CDT/ Conpes/Social/156.pdf

Fedesarrollo (2010) El sistema pensional en Colombia: retos y alternativas para aumentar la cobertura [Documento de trabajo]. Recuperado de http://www.fedesarrollo. org.co/wp-content/uploads/2011/08/El-sistema-pensional-en-Colombia_Retos-y-alternativas-para-aumentar-la-cobertura-12-de-abril-2011.pdf

Ley 1580 de 2012 (1 de octubre) Por la cual se crea la Pensión Familiar. Diario oficial no 48.570.

Ministerio de Trabajo (2015) Nuevo modelo de protección a la vejez. Recuperado dehttp:// www.mintrabajo.gov.co/pensiones.html

Ministerio de Trabajo, (marzo de 2015), Presentación del nuevo modelo de la protección de la vejez [Documento de trabajo]. Recuperado de .http://mintrabajo.gov.co

Pérez, V. Defensor del pueblo (2007) El derecho a la igualdad. Bogotá: Imprenta Nacional de Colombia 
Santa, M, (2010), El sistema pensional en Colombia Retos y Alternativas para aumentar la cobertura. Recuperado de http://www.repository.fedesarrollo.org.co/ bitstream/11445/351/1/El-sistema-pensional-en-Colombia_Retos-y-alternativas-para-aumentar-la-cobertura-12-de-abril-2011. pdf

Suárez, M. (2006) Principio de igualdad en la constitución y la jurisprudencia constitucional. Medellín: Biblioteca jurídica Jurídica Diké

Unidad de Gestión Pensional y Parafiscal UGPP (2015) Leyes sobre pensiones,. Recuperada de en http://www.ugpp.gov.co/pensiones/leyes-sobre-pensiones. 